\title{
CLIMA SOCIAL FAMILIAR Y ESTRÉS EN LOS PROFESORES DE EDUCACIÓN BÁSICA REGULAR DE LA PROVINCIA DE ILO, 2012.
}

\author{
Family and Social Climate Stress in Regular Basic Education Teachers in \\ the Province oflilo, 2012.
}

Nora Graciela Díaz Dueñas ${ }^{4}$

\section{RESUMEN}

Objetivo: Este estudio se propuso como objetivo establecer la relación que existe entre el clima familiar y el estrés en los profesores de Educación Básica Regular de la provincia de llo, 2012.

Método: El estudio se planteó como investigación de tipo correlacional con diseño no experimental transeccional correlacional. La población estuvo conformada por el conjunto de profesores del nivel inicial, primario y secundario de la provincia de llo, que asciende a 1122 personas. Y se trabajó con una muestra probabilística estratificado, conformada por 232 profesores. Como instrumentos de recolección de datos, se aplicaron la Escala de Clima Familiar de Moos, una escala de 90 ítems, que explora tres dimensiones: relaciones familiares, crecimiento personal y mantenimiento del sistema; y el Inventario para la evaluación del estrés individual, un inventario de 96 ítems que identifica el nivel de estrés en función de diferentes aspectos: estilo de vida, ambiente, síntomas, empleo / ocupación, relaciones y personalidad.

Resultados: Se encontró relación significativa entre el clima familiar y el estrés, pero con características que conviene apuntar. Primero, al analizar la posible relación entre las

\footnotetext{
${ }^{4}$ Magister en Docencia Universitaria y Gestión Educativa
}

variables, se encontró que existe correlación negativa media $($ Rho $=-$ 0,585 ) entre las puntuaciones que el docente alcanza en las escalas de medición de las variables clima familiar y estrés. $Y$ segundo, la correlación identificada es altamente significativa $(p<0,01)$. Estos datos evidencian una relación inversa entre las variables, lo que supone que mientras mayor es la puntuación alcanzada en la escala de clima familiar, menor es la puntuación alcanzada en la escala de estrés. En otras palabras, mientras más alto es el clima familiar que experimenta el docente, menor es el estrés que padece; y de la misma manera, mientras más bajo es el clima familia que rodea al docente, mayor es el estrés que experimenta en su vida.

Conclusiones: Como conclusión general, se encontró que existe una relación inversa entre el clima familiar y el estrés en los profesores de Educación Básica Regular de la provincia de Ilo, 2012, de modo tal que mientras mayor es el clima familiar, menor es el nivel de estrés que experimenta el profesor.

\section{PALABRAS CLAVES}

Clima Familiar, estrés, personalidad, estilo de vida, ambiente.

\section{ABSTRACT}

Objective: This study set the objective of establishing the relationship between the family environment and stress on teachers of Basic Education of the province of Ilo, 2012. 
Method: The study was planned as correlational research with correlational no experimental design. The population was conformed by all teachers in primary and secondary schools in the province of llo, amounting to 1122 people initial levels. And we worked with a stratified probability sample, consisting of 232 teachers. As data collection instruments, the Family Climate Scale Moos, a scale of 90 items, which explores three dimensions were applied: family relationships, personal growth and system maintenance; and Inventory for the assessment of individual stress, an inventory of 96 items identifying the stress level based on different aspects: lifestyle, environment, symptoms, employment / occupation, relationships and personality.

Results: significant relationship between family environment and stress, but with features worth noting was found. First, to analyze the possible relationship between the variables, it was found that there is negative correlation average (Rho = -0.585) between the scores achieved in the teacher rating scales of family climate variables and stress. And second, the identified correlation is highly significant $(p<0.01)$. These data show an inverse relationship between the variables, which means that the higher the score achieved on the scale of family environment, the lower the score achieved on the stress scale. In other words, the higher is the family environment experienced by the teacher, the lower the stress disorder; and in the same manner, the lower is the family atmosphere that surrounds the teaching, the greater the stress experienced in your life.

Conclusions: As a general
conclusion, we found an inverse
relationship between family
environment and stress on teachers
of Basic Education of the province of
llo, 2012, so that the higher the
family atmosphere, the lower the
level stress experienced by the
teacher.

\section{KEYWORDS}

Family climate, stress, personality, lifestyle, environment

\section{INTRODUCCIÓN}

Para nadie en el Perú es un secreto que la situación del sector educación dista mucho de lo que el discurso oficialista y las buenas intenciones de políticos y funcionarios manifiestan, y se encuentra radicalmente lejos de lo que se esperaría para un país en crecimiento económico sostenido y que empieza a comprender la necesidad de insertarse en el concierto de las naciones que aprovechan sus recursos humanos, cognoscitivos e intelectuales, más que los recursos naturales, para afianzar su crecimiento interno y el desarrollo de su población. En realidad, desde hace ya algunos años se viene sosteniendo que el Perú asiste a uno de los momentos más difíciles de su historia en lo que se refiere a Educación (Garcilaso, 2012). Por ello, durante la última década se han dado diferentes esfuerzos por parte de los gobiernos de turno para revertir esta situación; esfuerzos que empiezan por la introducción de la Ley General de Educación, Ley 28044, en el 2003, durante la presidencia de Alejandro Toledo; la activación de un proceso de evaluación docente que creó suspicacias y desató fuerte polémica durante el gobierno de Alan García; la introducción de la carrera pública magisterial, el 2007, que supone el esfuerzo de estimular a los docentes 
mejor capacitados y calificados para cumplir su función y misión; y hoy la introducción de la nueva Ley de Reforma Magisterial, que ya generó oposición.

Se trata de un clima social y político en el cual asumir la profesión docente entraña un reto que, por lo general, sobrepasa la actividad laboral del profesor y deriva en necesidades personales cada vez más amplias, que, tanto desde una perspectiva individual como social, no son suficientemente atendidas.

Por otro lado, las personas que se dedican a trabajar en lo que se ha denominado "profesiones asistenciales", en el marco del servicio público, como ocurre con los profesionales de la enseñanza (profesores de todos los niveles), tienden a sentirse fuertemente afectadas por una sensación de aburrimiento o hastío, que es común tanto en la insatisfacción laboral como en el síndrome de Burnout, y verse sometidas a un gran desgaste profesional (Ayuso, 2006). Estas profesiones exigen entrega; implicación, ciertos niveles de idealismo y un indudable servicio a los demás y si todo esto se asienta sobre una personalidad que se puede calificar de perfeccionista, con un alto grado de autoexigencia y con una gran tendencia a implicarse en el trabajo, la persona puede caer en una situación de desequilibrio entre sus expectativas individuales y la realidad del trabajo diario (Ayuso, 2006). En otras palabras, las profesiones asistenciales, y entre ellas, la docencia, pueden convertirse en actividades laborales que no sólo demandan esfuerzo, dedicación y compromiso por parte del trabajador, sino también niveles de estrés que pueden terminar afectando tanto a la persona, sus vínculos familiares, e incluso su desempeño.

Frente a ello, conseguir el equilibrio entre las demandas de los roles familiares y laborales constituye un reto diario para muchos trabajadores. Y si bien esto en sí no constituye una fuente de insatisfacción o malestar, pues afrontar distintos roles y retos puede ser una fuente de compensaciones, familiares, psicológicas, sociales y laborales con el correspondiente incremento del bienestar y la autoestima; en realidad, para muchas personas este reto supone una fuente de malestar y distrés que pueden desembocar en importantes desajustes personales y sociales (Pérez, López y De la Rosa, 2005).

Se ha constatado que en el trabajo de algunos colectivos es el estrés, tal vez, el problema que más atenta contra su salud, frente a otros factores de riesgo de origen material o ambiental (Nogareda, 2011). El estrés es, en la actualidad, uno de los problemas a los que se está prestando una atención creciente. Ello, debido fundamentalmente al conocimiento de la magnitud e importancia de sus consecuencias, aunque también por el progresivo conocimiento de este problema, de cómo evaluarlo, gestionarlo y prevenirlo (Nogareda, 2011).

En el caso de la profesión docente, no se debe desestimar el estado de opinión que existe entre los profesionales de la enseñanza y parte de la sociedad, sobre la dificultad que implica impartir clases a los adolescentes de hoy. Este clima de insatisfacción, recoge consideraciones negativas respecto a la falta de disciplina en el alumnado, falta de apoyo de padres y madres e incluso de la administración educativa, el importante número de bajas entre docentes por enfermedades mentales (depresión, ansiedad, etc.), determinadas rutinas perniciosas que se establecen en los centros y que no permiten el desarrollo óptimo de la actividad profesional (falta de profesorado de apoyo, inadecuación de horarios, las tutorías de las clases "más conflictivas" son asumidas por profesorado recién llegado al centro, etc.) (Ayuso, 2006). Todo esto y más, hace 
que la vivencia de su profesión por parte de muchos profesores y profesoras, no se haga de manera positiva, lo que los lleva a engrosar las filas de aquellos profesionales que sufren el día a día de su trabajo, llegando poco a poco a mayores cotas de insatisfacción y malestar docente (Ayuso, 2006).

Se debe reconocer que la sociedad actual vive un proceso de continuo cambio, producto de los avances de la industrialización y de la tecnología, los mismos que están provocando una crisis en las instituciones sociales que tradicionalmente se consideraban pilares básicos de la sociedad; entre ellas, la familia es quizá la más afectada. Hoy es frecuente que ambos padres trabajen fuera del hogar y que, como consecuencia, la vida familiar pierda intensidad, sobre todo por la falta de tiempo. Al mismo tiempo, el aumento de las tensiones familiares ha generado ya $-\mathrm{y}$ es probable que siga aumentando-, un alarmante número de separaciones formales o prácticas, y un incremento de la violencia familiar. Por otro lado, factores sociales como la búsqueda de status, la relajación moral, el efecto disociante de la televisión, la falta de preparación para el matrimonio, las responsabilidades con los hijos, traen como consecuencia perturbaciones en la personalidad, elevados índices de estrés, violencia social y en general una diversidad de problemas, a los cuales no están exentos los profesores de hoy.

En ese sentido, es de gran importancia que el profesor tenga metas bien definidas en cuanto a su plan de vida como persona y a su rol profesional, pues hacerlo le permitirá alcanzar su desarrollo social e individual. Un docente con una personalidad integrada y bien configurada $\mathrm{y}$, por ende, una valoración positiva tanto social como individual inciden en forma favorable en el desarrollo personal de sus alumnos, los que en forma consciente e inconsciente van construyendo su valor como persona según los estímulos que perciba y la actuación de su profesor. Pero si el educador no posee una personalidad bien integrada, una autoimagen positiva, un equilibrio emocional y un alto grado de estimación personal, no podrá ofrecer a sus alumnos una interacción humana y fecunda que les permita a su vez, con espíritu de superación, ir construyendo una imagen adecuada de sí mismos.

Aunque no son muchos los estudios que defiendan que el estrés en el colectivo docente de una institución, una comunidad o una ciudad, puede ser en cierta medida aminorado o controlado por el profesor que experimenta un clima familiar favorable, el hecho de que esta relación se haya observado en otros espacios laborales (Pérez, López y De la Rosa, 2005) o en otros sujetos (Moreno, Estévez, Murgui y Musitu, 2009), da lugar a considerar como válido el supuesto de que el clima familiar, entendido como una estructura natural en la cual se elabora pautas de interacción psicosociales que rigen el funcionamiento de los miembros de una familia, influye en el nivel de estrés que puede alcanzar un profesor, en el entendido de que un clima familiar favorable tenderá a reducir los niveles de estrés negativo en el profesor.

\section{MATERIALES Y MÉTODO}

Por su finalidad, la investigación es básica. De acuerdo al problema y tipo de conocimiento a lograr, el diseño de investigación es no experimental transeccional correlacional (Hernández et al., 2006). El estudio se desarrolló en la provincia de Ilo, Región Moquegua, que comprende los distritos de Ilo, Pacocha y Algarrobal. El tiempo corresponde al año 2012. 
La población está conformada por los profesores de los niveles inicial, primaria y secundaria de la provincia de llo, que hace un total de $N=1,122$. El tamaño de la muestra es de 232 , se calculó por estimación de proporciones para poblaciones finitas. Para un nivel de confianza del 95\%. Los elementos de muestra se seleccionaron mediante el procedimiento conocido como tómbola o rifa.

La Escala de Clima Familiar de Moos es una escala de 90 ítems, con alternativas de respuesta Verdadero - Falso, de los cuales 54 tienen respuesta verdadera y 36 falsa. El instrumento explora tres dimensiones: relaciones familiares, crecimiento personal y mantenimiento del sistema. La dimensión relaciones familiares está constituida por tres sub escalas: cohesión, expresividad y conflicto. La dimensión crecimiento personal está constituida por cinco sub escalas: autonomía, orientación al logro, orientación intelectual, orientación recreacional y orientación moral. La dimensión mantenimiento del sistema está constituida por dos sub escalas: organización y control.

El procesamiento de datos se hizo de forma automatizada con la utilización de medios informáticos. Para ello, se utilizaron el soporte informático SPSS 19, paquete con recursos para el análisis descriptivo de las variables y para el cálculo de medidas inferenciales; y Excel, paquete de Microsoft Office que cuenta con potentes recursos gráficos y funciones específicas para el ordenamiento de datos.

En cuanto a estadísticos, se hizo uso de tablas de frecuencia simples, para la presentación de las variables en forma de frecuencias simples y porcentuales; gráficos circulares, para visualizar las categorías en ambos grupos de población.

Además, se utilizó el coeficiente de correlación por rangos de Spearman (Rho), que es una prueba no paramétrica cuando se desea medir la relación entre dos variables y no se cumple el supuesto de normalidad en la distribución de tales valores. Se designa por $r_{s} \circ \rho$. Al igual que el coeficiente de correlación de Pearson, este coeficiente reúne dos propiedades que identifican la naturaleza de una relación entre dos variables: su signo, que puede ser positivo o negativo; y su magnitud. El coeficiente de correlación varía de $-1 a+1$. El valor de $r=1$ muestra una correlación lineal positiva perfecta entre dos variables $\mathrm{X}$ e $\mathrm{Y}$. Por el contrario, un valor de $r=-1$ indica una correlación lineal negativa perfecta entre dos variables $\mathrm{X}$ e $\mathrm{Y}$. Pero si el valor de $r$ es igual a 0 , las dos variables $\mathrm{X}$ e $\mathrm{Y}$ no están correlacionadas. (Lopes, 2000)

\section{RESULTADOS}

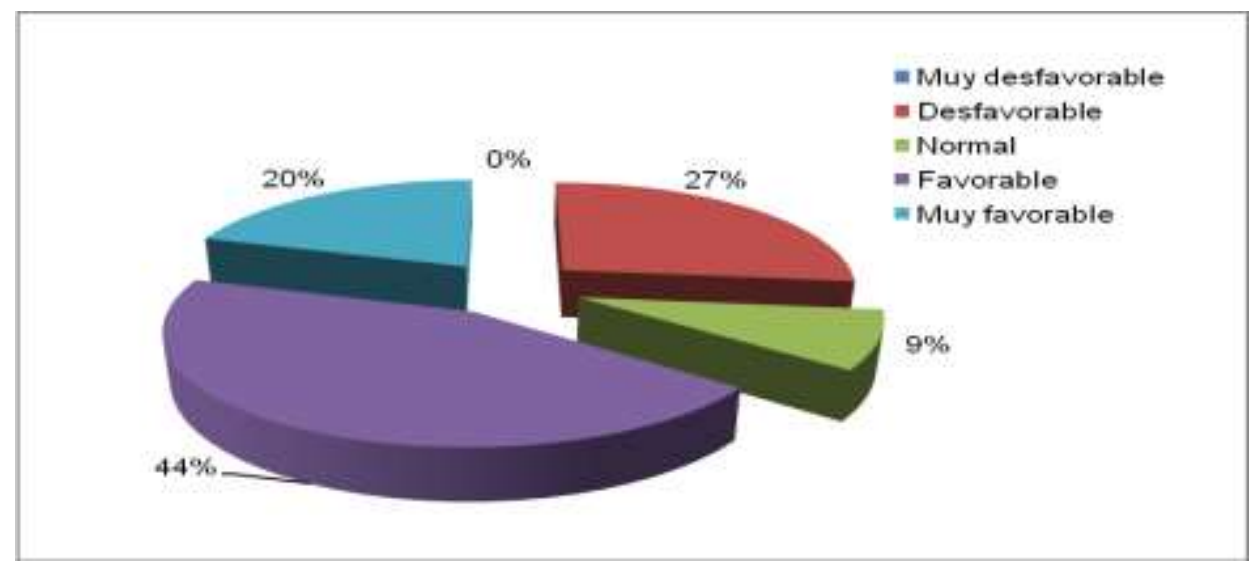

Fuente: Tabla $\mathrm{N}^{\circ} 2$ 
Figura № 1

Profesores por clima familiar. Provincia de Ilo, 2012.

En la figura № 1 se aprecia la distribución de profesores en función del clima familiar que manifiestan. Se encontró una distribución que evidencia un clima familiar favorable, con una mayoría relativa conformada por 103 profesores, que representan el $44,4 \%$ del conjunto, que se distribuyen en esta categoría de la variable, y 47 profesores, que representan la quinta parte del conjunto, que evidencian un clima familiar muy favorable. En contraste, 62 profesores, que representan el 26,7\%, se distribuyen en la categoría de clima familiar desfavorable, mientras que 20 profesores, que representan el $8,6 \%$ del conjunto, lo hacen en la categoría de clima familiar normal.

Las cifras muestran que se trata de un grupo humano que experimenta un clima familiar favorable o muy favorable, con categorías que sobrepasan lo esperado en términos normales.

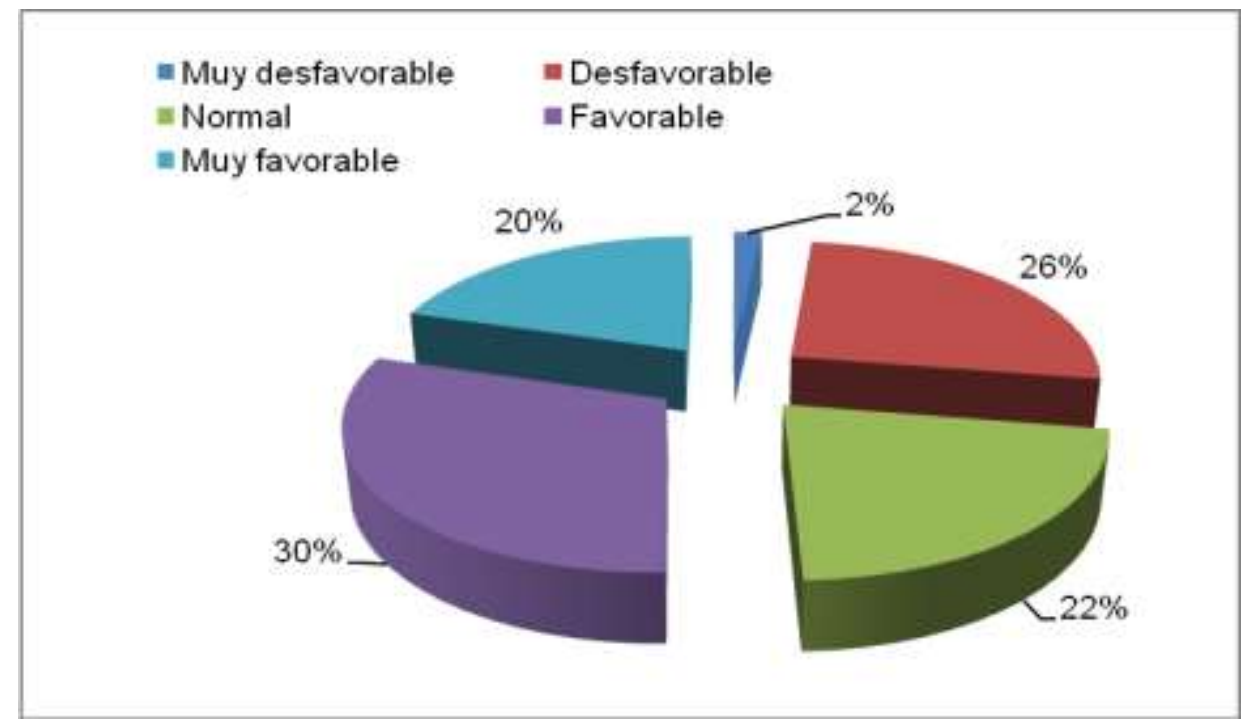

Fuente: Tabla $N^{\circ} 4$

Figura № 2

Profesores por clima familiar: relaciones familiares. Provincia de Ilo, 2012.

En la figura $\mathrm{N}^{\circ} 2$, en lo que respecta a la primera dimensión del clima familiar, relaciones familiares, se encontró predominio conjunto de los climas familiares favorable y muy favorable. Estas categorías, agrupan al 50,5\% del grupo, con 70 profesores, que representan el $30,2 \%$ y alcanzan el nivel favorable, y 47 profesores que representan el $20,3 \%$ y alcanzan el nivel muy favorable. En el nivel normal, se ubican 51 profesores, que representan apenas el $22 \%$ del conjunto, que es una cifra y proporción menor que la que corresponde al nivel desfavorable, donde aparecen 60 personas, que representan el 25,9\%. En la categoría muy desfavorable aparecen sólo cuatro profesores, que representan apenas el $1,7 \%$ del conjunto de profesores muestreados. 
De acuerdo a estos resultados, las relaciones familiares que experimentan los docentes, como el primer aspecto que define el clima familiar, se caracterizan por ser favorables para el profesor.

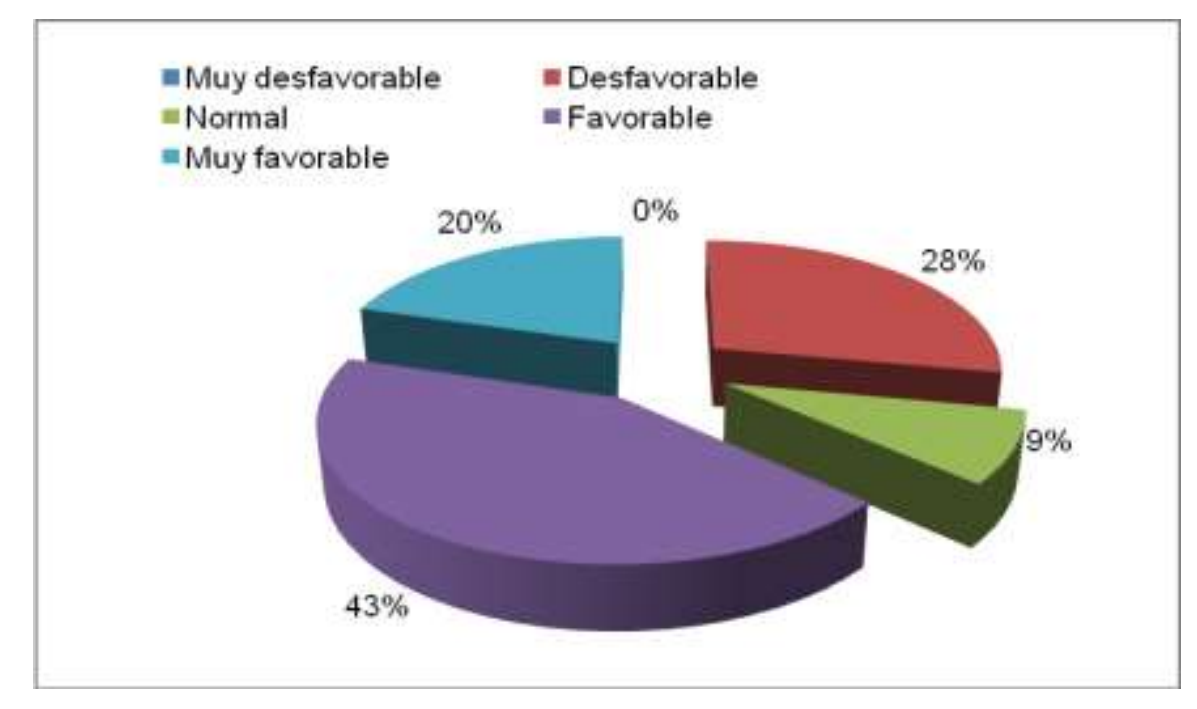

Fuente: Tabla $N^{\circ} 6$

Figura № 3

Profesores por clima familiar: crecimiento personal. Provincia de Ilo, 2012.

En la figura $\mathrm{N}^{\circ} 3$ se aprecia la distribución de profesores en función de la segunda dimensión del clima familiar: crecimiento personal. Se observa una mayoría relativa conformada por 103 profesores, que representan el 43,1\% del conjunto y se distribuyen en la categoría de clima familiar favorable, y 47 profesores, que representan el $20,3 \%$ del total, en la categoría muy favorable. Cada una de estas dos categorías supone familias cuyo funcionamiento familiar supera lo esperado en términos normales, expresado por la categoría de clima familiar normal, que apenas congrega el $8,6 \%$ de total. En contraste, en la categoría de clima familiar desfavorable se ubican 65 profesores, que representan el $28 \%$ del conjunto.

En síntesis, predominan las categorías de clima familiar favorable, en los cuales el clima familiar que experimenta el docente favorece la importancia que se asigna y atribuye en la familia a aquellos procesos que contribuyen a su desarrollo personal. 


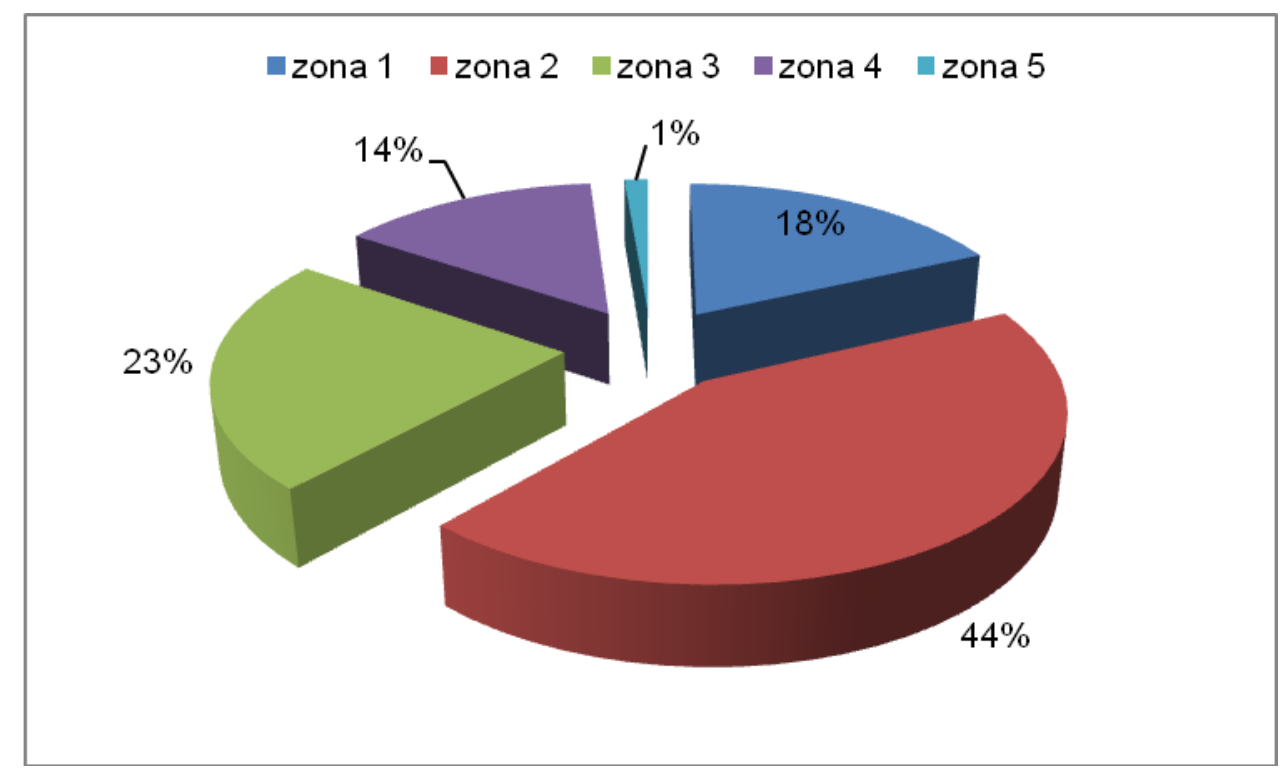

Fuente: Tabla $N^{\circ} 8$

Figura № 4

Profesores por estrés. Provincia de llo, 2012.

En la figura $\mathrm{N}^{\circ} 4$ se presenta la información recogida en torno al estrés que evidencian los profesores de acuerdo a la escala utilizada. En la zona 2, nivel bajo de estrés, se ubican 101 profesores, que representan el $43,5 \%$ del grupo, y en la zona 1, 42 profesores, que representan el 18,1\% del grupo. En el nivel normal se distribuyen 53 personas, que representan el 22,8\%, mientras que en las zonas de alto estrés, se reúne poco menos del 16\% de profesores, con 33 casos $(14,2 \%)$ para la zona 4 , y tres casos $(1,3 \%)$ para la zona 5 , que indica estrés peligroso para la persona. Es claro el predominio conjunto de los niveles bajos de estrés, zona 1 y zona 2, que indican un nivel peligrosamente pobre de estrés y un nivel bajo de estrés, respectivamente. Esto muestra que, en términos generales, los profesores no están experimentado mayores niveles de estrés, lo que entraña un bajo nivel de preocupación por las diferentes actividades que realizan.

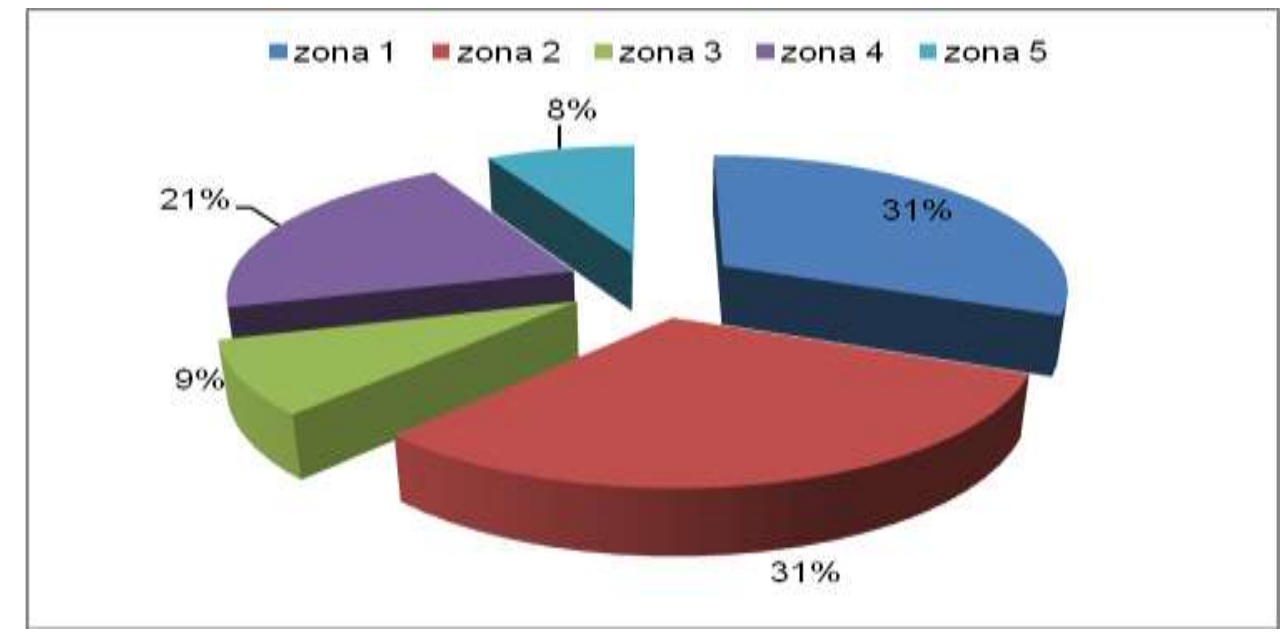

Fuente: Tabla $N^{\circ} 10$ 


\section{Figura № 5}

Profesores por estrés: estilo de vida. Provincia de Ilo, 2012.

En la figura $N^{\circ} 5$ se presenta información en torno a la primera dimensión del estrés, estilo de vida. En cada caso, se identifican 72 profesores, que representan $31 \%$ del grupo. Frente a estas dos categorías, en el nivel normal se distribuyen sólo 20 personas, que representan el 8,6\% del grupo, mientras que en las zonas de alto estrés, en este caso, se reúne casi $30 \%$ de profesores, con 49 casos $(21,1 \%)$ para la zona 4 , y 19 casos $(8,2 \%)$ para la zona 5 . Se observa, entonces, predominio conjunto de los niveles bajos de estrés, zona 1 y zona 2, que indican un nivel peligrosamente pobre de estrés y un nivel bajo de estrés, respectivamente. En otras palabras, no hay mayor preocupación de los docentes por el estilo de vida o, a modo de corolario, están de alguna manera conformes con el estilo de vida que llevan.

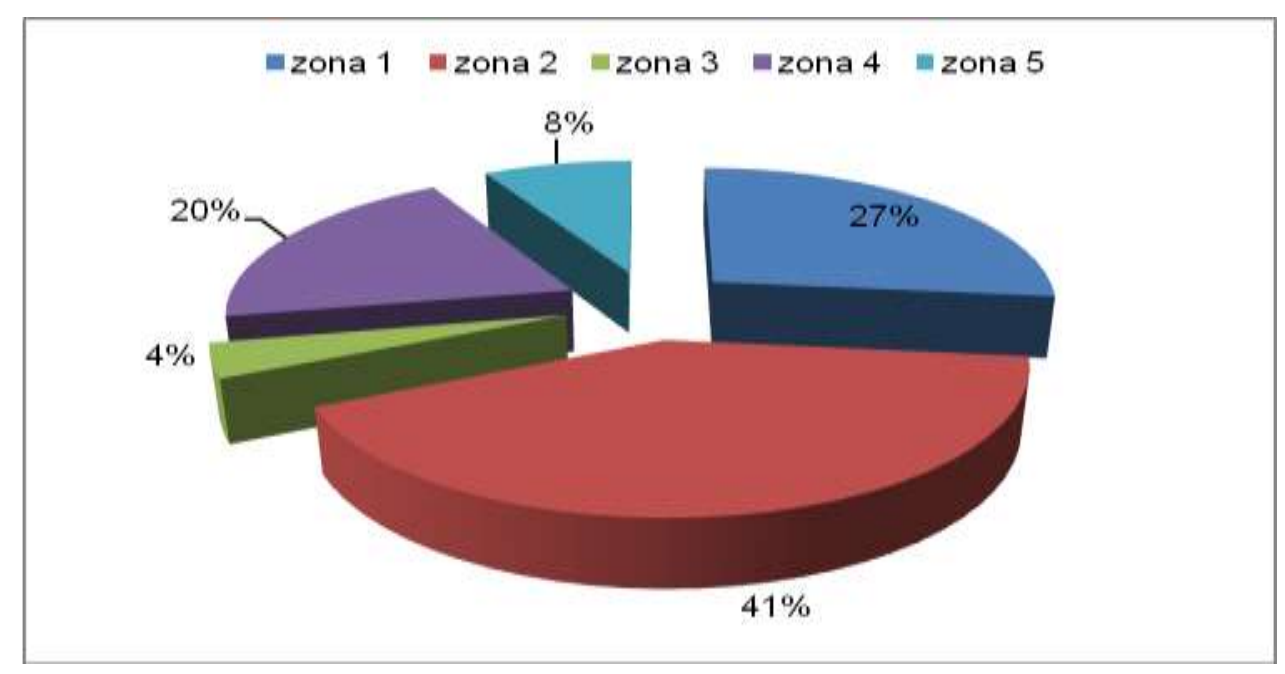

Fuente: Tabla 12

Figura № 6

Profesores por estrés: ambiente. Provincia de llo, 2012.

En la figura $N^{\circ} 6$ se presenta información en torno a la segunda dimensión del estrés: ambiente. Se identifican 96 profesores, que representan $41,4 \%$ del grupo, para la zona 2; y 62 profesores, que representan $26,7 \%$ del grupo, para la zona 1. Frente a estas dos categorías, en el nivel normal se distribuyen sólo nueve personas, que representan apenas el 3,9\% del grupo, mientras que en las zonas de alto estrés, se reúne $28 \%$ de profesores, con 46 casos $(19,8 \%)$ para la zona 4 , y 19 casos $(8,2 \%)$ para la zona 5 .

Se observa, en consecuencia, marcado predominio conjunto de los niveles bajos de estrés, zona 1 y zona 2, donde se reúne $68 \%$ de profesores. Esto significa que, en términos generales, el ambiente que rodea al docente no genera en ellos niveles de estrés ni siquiera normales. 


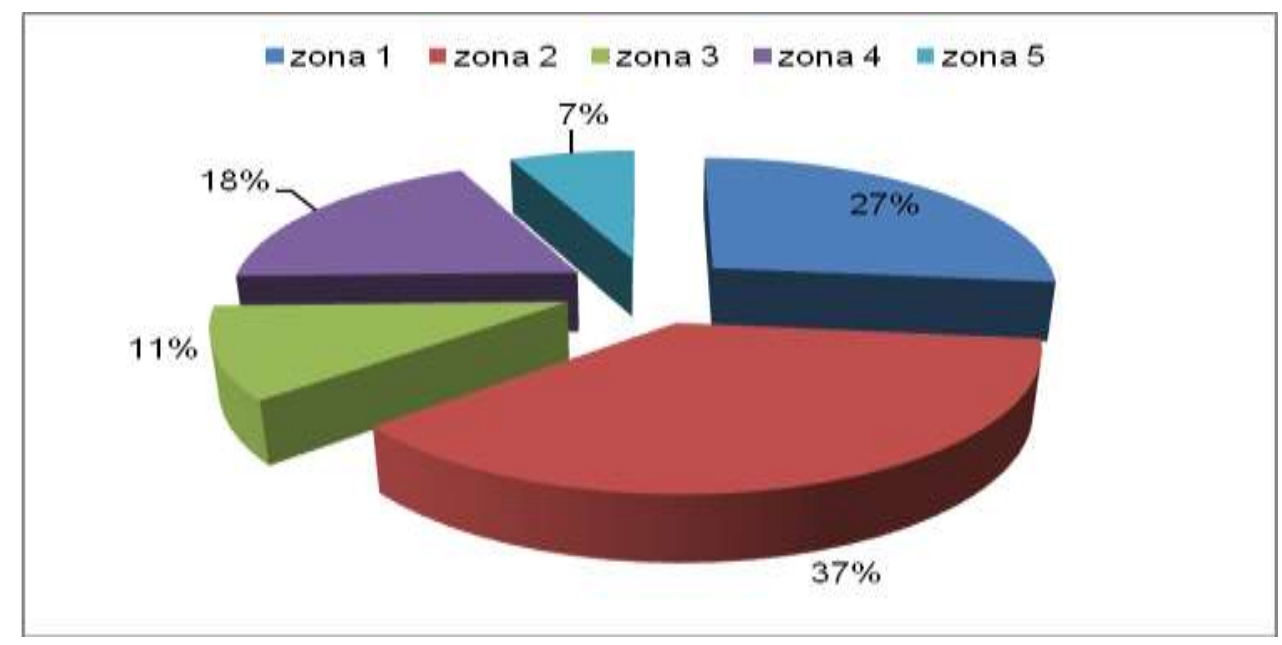

Fuente: Tabla 14

Figura № 7.

Profesores por estrés: trabajo. Provincia de Ilo, 2012.

En la figura $\mathrm{N}^{\circ} 7$ se presenta información en torno a la cuarta dimensión del estrés: trabajo. Al igual que en los casos anteriores, se observa predominio conjunto de los niveles bajos de estrés, zona 1 y zona 2. En este caso, estos dos niveles agrupan $63,8 \%$ de profesores. $Y$ se identifican 86 profesores, que representan $37,1 \%$ del grupo, en la zona 2; y 62 profesores, que representan $26,7 \%$ del grupo, en la zona 1. Por otro lado, en el nivel normal se distribuyen 25 profesores, que representan el $10,8 \%$ del grupo; mientras que en las zonas de alto estrés, se reúne nuevamente un $25 \%$ de profesores, con 43 casos $(18,5 \%)$ para la zona 4 , y 16 casos $(6,9 \%)$ para la zona 5 .

Por increíble que parezca, el trabajo sólo constituye motivo de estrés para menos del $30 \%$ de docentes; en contraste, para más de la mitad del grupo, el trabajo no constituye motivo de preocupación que llegue a ocasionar estrés.

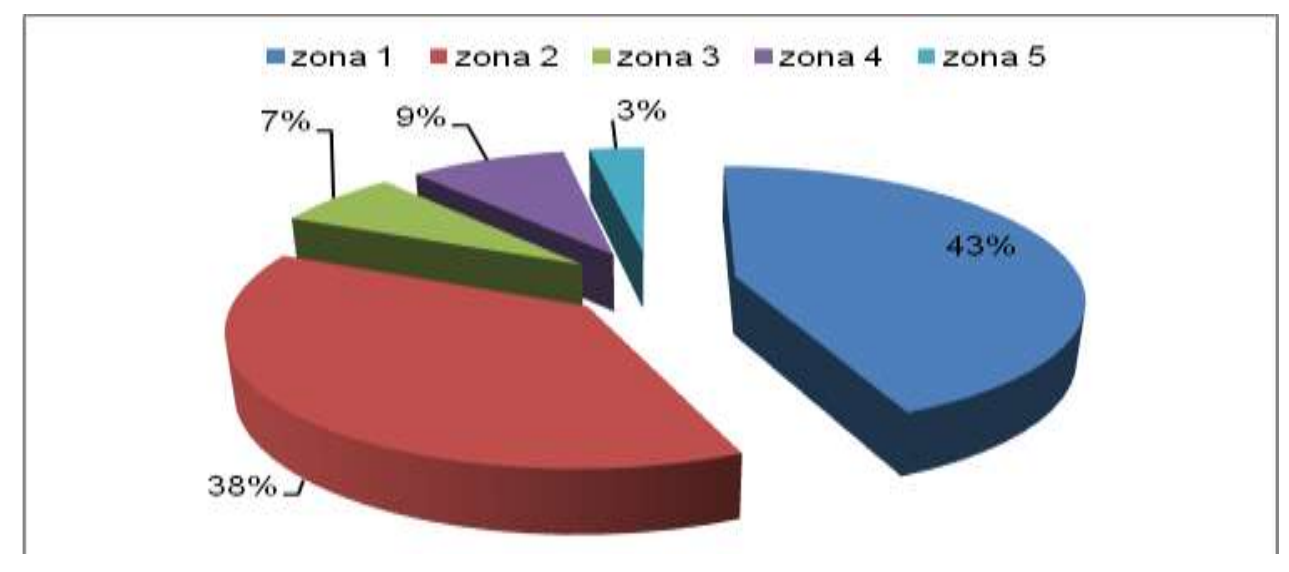

Fuente: Tabla 16

Figura № 8.

Profesores por estrés: personalidad. Provincia de Ilo, 2012. 
En la figura $N^{\circ} 8$ se presenta información en torno a la última dimensión del estrés: personalidad. Cabe señalar que el $81,4 \%$ del conjunto de profesores, con 101 profesores, que representan $43,5 \%$ del grupo, en la zona 1, y 88 profesores, que representan $37,9 \%$ del grupo, en la zona 2 . Por otro lado, en el nivel normal se distribuyen sólo 16 profesores, que representan apenas el 6,9\% del grupo; mientras que en las zonas de alto estrés, se agrupa esta vez sólo $11,6 \%$ del conjunto, con 20 casos (8,6\%) para la zona 4 , y siete casos (3\%) para la zona 5. En este caso, el predominio conjunto de los niveles bajos de estrés, zona 1 y zona 2, es bastante acentuado, pero se observa una variación importante respecto de las dimensiones anteriores; en este caso, la mayor frecuencia recae en el nivel 1. De todos modos, la valoración que el docente hace de sus propias cualidades, virtudes y defectos personales, así como de los hábitos de relación que pone en práctica en su interacción con los demás, no constituye un motivo que pudiera ocasionarle estrés.

Prueba estadística de la relación entre clima familiar y estrés

\section{Correlaciones}

\begin{tabular}{|c|c|c|c|c|}
\hline & & & $\begin{array}{c}\text { clima } \\
\text { familiar }\end{array}$ & estrés \\
\hline \multirow{6}{*}{$\begin{array}{l}\text { Rho de } \\
\text { Spearman }\end{array}$} & \multirow[t]{3}{*}{ ima familiar } & Coeficiente de correlación & 1,000 &,$- 585^{\star \star}$ \\
\hline & & Sig. (bilateral) & . & ,000 \\
\hline & & $\mathrm{N}$ & 232 & 232 \\
\hline & \multirow[t]{3}{*}{ estrés } & Coeficiente de correlación &,$- 585^{\star *}$ & 1,000 \\
\hline & & Sig. (bilateral) & ,000 & . \\
\hline & & $\mathrm{N}$ & 232 & 232 \\
\hline
\end{tabular}

**. La correlación es significativa al nivel 0,01 (bilateral).

Fuente: elaboración propia.

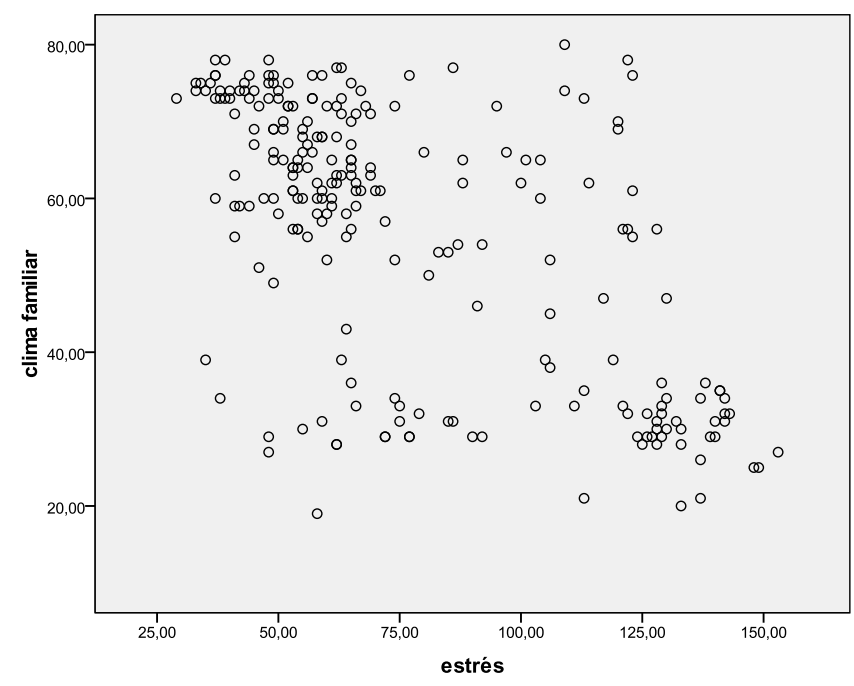

Fuente: Tabla 18 


\section{Figura № 9. \\ Gráfico de dispersión para estrés según clima familiar. Provincia de llo, 2012.}

Como se observa en la tabla, el análisis efectuado para probar la existencia de una relación entre las variables, demuestra que existe correlación negativa media (Rho $=-0,585$ ) entre las puntuaciones del docente en las variables clima familiar y estrés. La correlación es altamente significativa $(p=0,000)$. En otras palabras, se verifica una relación inversa entre las variables, lo que quiere decir que mientras mayor es la puntuación alcanzada en la escala de clima familiar, menor es la puntuación alcanzada en la escala de estrés. Dicho en términos sencillos, mientras más alto es el clima familiar, menor es el estrés que experimenta el docente; y por el contrario, mientras más bajos son los valores del clima familiar, mayor es el estrés. En el gráfico que se adjunta, se aprecia la distribución de puntos según los valores alcanzados en las dos variables.

\section{DISCUSIÓN}

En forma general, se encontró evidencia de una percepción favorable del clima familiar, en donde una mayoría de poco más del 65\% alcanza como mínimo el nivel alto. Para efectos de una apreciación comparada de las tres dimensiones de la variable, se encontró que, en términos relativos, la proporción de puntuación máxima alcanzada en las dimensiones son muy cercanas entre sí, lo que evidencia una notable consistencia en la forma como perciben el clima familiar. En ese sentido, para relaciones familiares, la proporción de puntuación máxima posible fue de $62,3 \%$; para crecimiento personal, $61,8 \%$; y para mantenimiento del sistema, $60,7 \%$.

En lo que concierne al estrés que evidencian los profesores, considerando la escala utilizada, es notorio el predominio de los niveles bajos de estrés, zona 1 y zona 2, que indican un nivel peligrosamente pobre y un nivel bajo de estrés, respectivamente. Más de la mitad del conjunto de profesores se distribuye en estas dos categorías, 43,5\% en la zona del bajo de estrés (zona 2), y 18,1\% en la zona de estrés peligrosamente pobre (zona 1).

En respuesta al objetivo general que se propuso este estudio, se encontró relación significativa entre el clima familiar y el estrés, pero con características que conviene apuntar. Primero, al analizar la posible relación entre las variables, se encontró que existe correlación negativa media (Rho $=-0,585)$ entre las puntuaciones que el docente alcanza en las escalas de medición de las variables clima familiar y estrés. Y segundo, la correlación identificada es altamente significativa $(p<0,01)$. Estos datos evidencian una relación inversa entre las variables, lo que supone que mientras mayor es la puntuación alcanzada en la escala de clima familiar, menor es la puntuación alcanzada en la escala de estrés. En otras palabras, mientras más alto es el clima familiar que experimenta el docente, menor es el estrés que padece; y de la misma manera, mientras más bajo es el clima familia que rodea al docente, mayor es el estrés que experimenta en su vida. 


\section{CONCLUSIONES}

a) Como conclusión general, se encontró que existe una relación inversa entre el clima familiar y el estrés en los profesores de Educación Básica Regular de la provincia de llo, 2012 (Rho = -0,585; $p=0,000$ ). En ese sentido, mientras más altas son las puntuaciones alcanzadas por el profesor en la Escala de Clima Familiar de Moos, más bajas son las puntuaciones que alcanza en el Inventario para la evaluación del estrés individual, lo que implica que los desajustes que se experimentan a nivel de clima de la familia constituyen un factor de especial relevancia en el estrés que experimenta el profesor.

b) El nivel de clima familiar en los profesores de Educación Básica Regular de la provincia de Ilo, 2012, es favorable; en ese sentido, 44,4\% de docentes se ubica en esta categoría, lo cual entraña un nivel de interacciones que se producen en su grupo familiar que le permite estrechar sus vínculos, en un ambiente sano y enriquecedor.

c) La zona de estrés en los profesores de Educación Básica Regular de la provincia de Ilo, 2012, corresponde a estrés bajo (zona 2); en ese sentido, $43,5 \%$ de docentes se ubica en esta categoría, que entraña para el profesor una situación saludable y libre de riesgos, pero que también puede indicar un rendimiento por debajo de su capacidad.

\section{REFERENCIAS BIBLIOGRÁFÍCAS}

- ALVAREZ, B. \& Majmudar, J. (2001). ¿Quién está preparando a nuestros hijos para el siglo del conocimiento? Human Development Department LCSHD Paper Series No. 67S. Recuperado el 20 de noviembre de 2012, de:

http://www.oei.es/docentes/articulos/quien_preparando_nuestros_hijos_B M.pdf

- ARellano, R. (2000). Los estilos de vida en el Perú. Lima: Consumidores y Mercados.

- AREllano, R. (2003). Marketing. Enfoque América Latina. México, Mc Graw - Hill / Interamericana Editores.

- ARÓN, A.M. y Milicic, N. (2002). Desgaste profesional de los profesores y clima social escolar. Recuperado el 20 de noviembre de 2012, de: http://www.buentrato.cl/pdf/est_inv/desgas/dpa_aron1.pdf

- ÁVAlos, B., CARLSON, B. \& Aylwin, P. (2004). La inserción de profesores neófitos en el sistema educativo: ¿cuánto sienten que saben y cómo perciben su capacidad docente en relación con las tareas de enseñanza asignadas? Concurso Nacional de Proyectos Fondecyt Regular 2002. Recuperado el 20 de noviembre de 2012, de: http://www.eclac.org/ddpe/publicaciones/sinsigla/xml/7/19597/inserprofe.p df 
- AYUSO, J.A. (2006). Profesión docente y estrés laboral: una aproximación a los conceptos de Estrés Laboral y Burnout. Revista Iberoamericana de Educación, Vol. 39, №. 3, 1-14. Recuperado el 20 de noviembre de http://www.rieoei.org/deloslectores/1341 Ayuso.pdf de:

- BALDEON, N. (2006). Relación entre el Clima Social Familiar y nivel de Autoestima de los pacientes con tuberculosis que asisten al Centro Materno Infantil tablada de Lurín - Villa María del Triunfo. Lima, Perú.

- BELLO, M. \& Villarán, V. (2004). Educación, reformas y equidad en los países del los Andes y Cono Sur: Dos escenarios en el Perú. Buenos Aires: Instituto Internacional de Planeamiento Educativo (IIPE).

- CABALLERO, A. (2003). Factores que influyen como motivantes para un buen desempeño laboral en los docentes de una escuela del nivel medio superior. Tesis para optar el grado de maestro en Ciencias de la Administración con mención en Relaciones Industriales. Universidad.

Recibido: 28/5/2015

Aceptado para publicación:

$4 / 7 / 2015$ 